\title{
Upper extremity surface electromyography signal changes after laparoscopic training
}

\author{
Michal M. Nowakowski ${ }^{1}$, Paulina Trybek ${ }^{2}$, Mateusz Rubinkiewicz $^{3}$, Tomasz Cegielny ${ }^{1}$, Michał Romaniszyn $^{1}$, \\ Michał Pędziwiatr ${ }^{3,4}$, Łukasz Machura² \\ ${ }^{1}$ Department of Medical Education, Jagiellonian University Medical College, Krakow, Poland \\ ${ }^{2}$ Department of Computational Physics and Electronics, Institute of Physics, University of Silesia, Katowice, Poland \\ ${ }^{3} 2^{\text {nd }}$ Department of General Surgery, Jagiellonian University Medical College, Krakow, Poland \\ ${ }^{4}$ Center for Research, Training and Innovation in Surgery (CERTAIN Surgery), Krakow, Poland
}

Videosurgery Miniinv 2018; 13 (4): 485-493 DOI: https://doi.org/10.5114/wiitm.2018.78744

\begin{abstract}
Introduction: Objective measures of laparoscopic skill in training are lacking.

Aim: To evaluate the changes in the surface electromyography (SEMG) signal during laparoscopic training, and to link them to intracorporeal knot tying.

Material and methods: Ten right-handed medical students (6 female), aged $25 \pm 0.98$, without training in laparoscopy, were enrolled in the study. With no additional training, they tied intracorporeal single knots for 15 min. Then underwent laparoscopic training and redid the knot tying exercise. During both events, SEMG was recorded from 8 measurement points on the upper extremities and neck bilaterally. We analyzed changes in sEMG resulting from training and tried to find SEMG predictive parameters for higher technical competence defined by the number of knots tied after the training.

Results: The average number of knots increased after the training. Significant decreases in activity after the training were visible for the non-dominant hand deltoid and trapezius muscles. Dominant and non-dominant hands had different activation patterns. Differences largely disappeared after the training. All muscles, except for the dominant forearm and non-dominant thenar, produced a negative correlation between their activities and the number of tied knots. The strongest anticorrelation occurred for the non-dominant deltoid $(r=-0.863$, $p$ <0.05). Relatively strong relationships were identified in the case of the non-dominant trapezius and forearm muscles $(r=-0.587, r=-0.504)$.

Conclusions: At least for some muscle groups there is a change in activation patterns after laparoscopic training. Proximal muscle groups tend to become more relaxed and the distal ones become more active. Changes in the non-dominant hand are more pronounced than in the dominant hand.
\end{abstract}

Key words: laparoscopy, assessment, training, surface electromyography.

\section{Introduction}

The introduction of laparoscopy to surgery shifted many paradigms, some of them concerning surgical education. One of the most significant was the possibility or perhaps even the necessity to develop technical/manual skills in a simulated environment. Because of the very long learning curve, "box trainers" became commonplace in many surgical wards around the world [1-3]. There is evidence that the

\section{Address for correspondence}

Michał Pędziwiatr MD, PhD, $2^{\text {nd }}$ Department of General Surgery, Jagiellonian University Medical College, 21 Kopernika St, $31-501$ Krakow,

Poland, phone: +48 6085523 23, e-mail: michal.pedziwiatr@uj.edu.pl 
learning curve can be influenced by ex vivo training [4]. Despite the efforts to shorten the learning curve with novel technical solutions, extensive practice is still necessary [5]. Nowadays, every surgical trainee should follow the path of learning, starting with necessary knowledge followed by observation, training in a simulated environment of various degrees of fidelity, and finishing with supervised practice in real clinical work [6]. A few validated training protocols are available to choose from based on personal preferences and equipment at hand [7-9]. Despite the fact that laparoscopic simulators have been present on the market for a long time, proper assessment methodologies are still under development [10]. Even the most widespread training tools lack proper validity and discriminatory value to be accepted as assessment tools without further enquiry [11]. Some rudimentary benchmarks have been developed, usually based on the quantitative assessment. They include, but are not limited to, numbers of intracorporeal knots tied or elements transferred in a given period of time [12, 13]. Previously, simulation-based training was available only in selected, specialized centers. Recent developments and the introduction of simulation-based training to all medical schools in Poland has increased the access to that type of equipment for surgical trainees. Higher accessibility requires even more that valid assessment strategies be developed.

Studies suggest that the difficulty of a task increases motor cortex excitability, which in turn may lead to extensive motor unit activation and lower precision resulting from larger portions of muscles activated [14]. Taking all that into account, one may speculate that as the task becomes less demanding, the activation of the motor cortex and

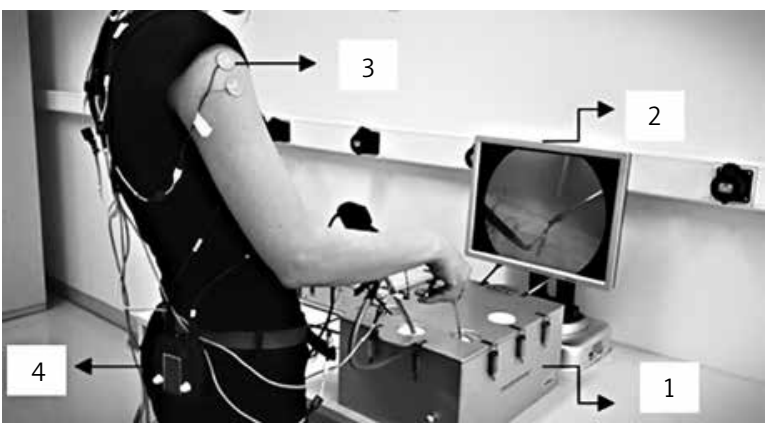

Photo 1. Equipment setup. 1 - box trainer, 2 computer monitor, 3 - electrodes and 4 - data recorder the motor unit recruitment resulting from it will also become lower. If that observation is confirmed, the measurement of activation patterns could be utilized to monitor the increase in trainee familiarization with the task or perhaps even some degree of competence, especially if combined with performance benchmarks.

Neuromuscular activation patterns can be effectively measured for various skeletal muscles by means of surface electromyography (sEMG) $[15,16]$. The general principle of this methodology is based on the recording of the bioelectrical signal as it propagates through the muscle. Activation patterns can be evaluated and characterized based on their global parameters, such as amplitude and frequency [17-19]. Alternatively, motor unit action potentials for individual motor units can be identified and tracked through the body of the muscle [17-20].

Since the SEMG signal is relatively easy to measure and, as indicated by the available literature, it could possibly be linked at least to the familiarization of a particular skill, we decided to combine laparoscopic training and SEMG recording in search for a correlation between the SEMG signal and traditional performance assessment.

\section{Aim}

Our aim was to evaluate the changes in the SEMG signal, occurring as a result of laparoscopic training, and to link them to laparoscopic skill acquisition measured by intracorporeal knot tying.

\section{Material and methods}

Ten right-handed medical students ( 6 female and 4 male) in the age range of 24-27 (25 \pm 0.98$)$ and without any previous training in laparoscopy were voluntarily enrolled in the study.

We used a standard laparoscopic box trainer with a high definition video camera and computer monitor. The complete equipment setup is depicted in Photo 1.

Students were asked to tie as many intracorporeal single knots as they could within 15 min: in the first step before and in the second step after structured laparoscopic training. The number of knots tied was recorded during each of those steps. This kind of exercise was chosen since it is often considered to be a good measure of laparoscopic technical competence [21]. 
Simultaneously, sEMG measurements were performed using a portable 8-channel sEMG data logger (OTBioelettronica, Torino, Italy) for sEMG data collection. Each time, four measurement points were used on each upper extremity. Concentric electrodes were applied bilaterally at the thenar eminence, proximal forearm, deltoid muscle and trapezius muscle, which represent index localizations proposed in the 2010 Consensus Statement by the International Society of Electrophysiology and Kinesiology [22]. The placement of electrodes is depicted in Photo 2.
The sampling frequency was set at $125 \mathrm{~ms}$. Each subject underwent measurement on two occasions; eight measurement points were used.

For calibration and reference purposes, each session consisted of baseline recording of relaxed muscles followed by data acquisition in maximal contraction. Calibration periods were later excluded from the analysis.

Before taking part in this experiment, all students completed a 2-day suturing course (total of $10 \mathrm{~h}$ ) and were familiar with the knot-tying technique in open surgery. They were also provided with stan-
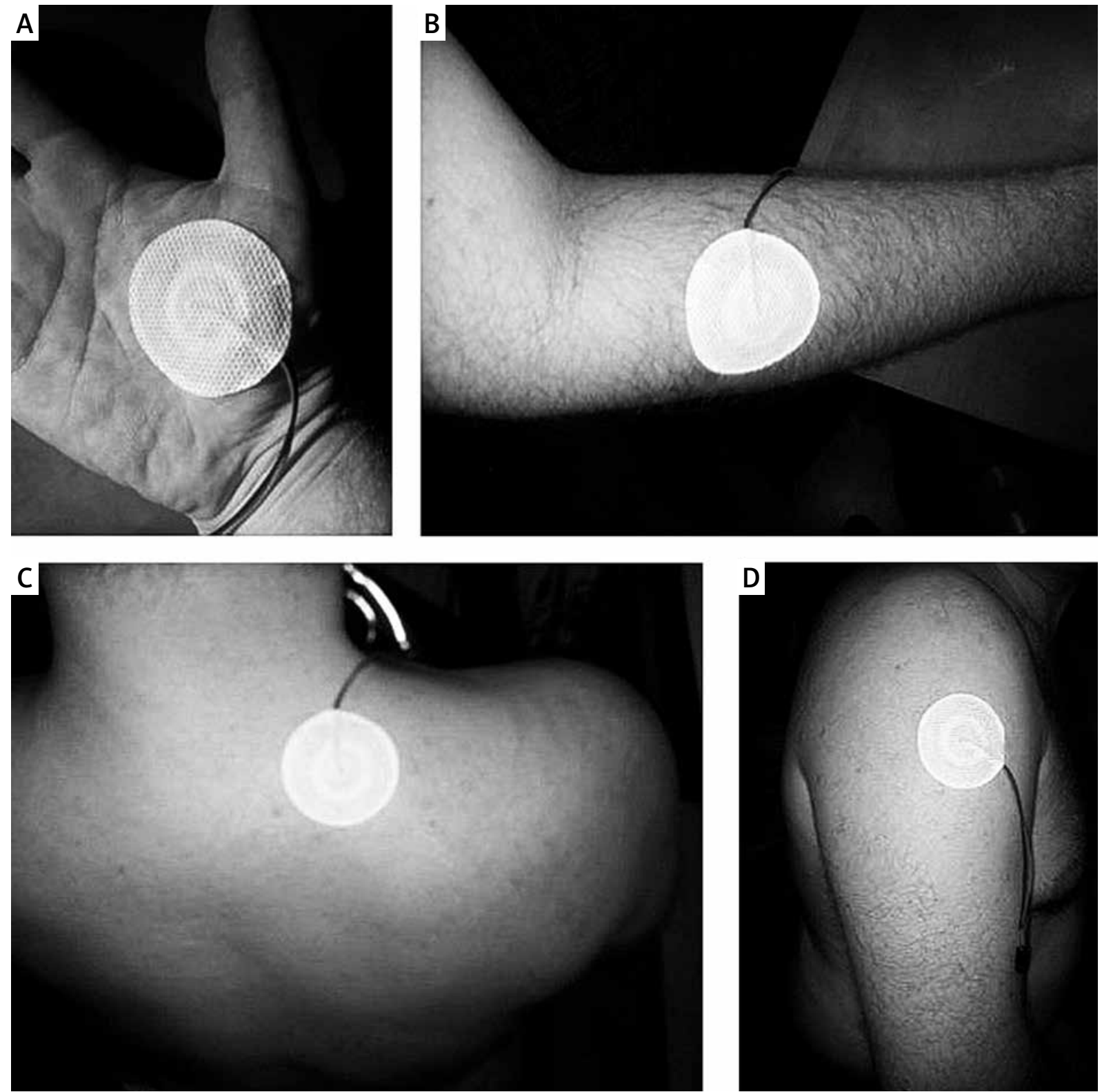

Photo 2. Electrode placement: $\mathbf{A}$ - thenar eminence, $\mathbf{B}$ - forearm, $\mathbf{C}$ - trapezius muscle, $\mathbf{D}$ - deltoid 
dardized information on the aims of the exercise and confirmed their understanding. Students had not received any previous training in laparoscopic knot tying.

After initial measurements, all participants received laparoscopic skills training according to a standardized procedure.

All students were trained in one-on-one fashion by two consultant laparoscopic surgeons with extensive experience in simulator-based minimally invasive surgery training. Since the literature confirms that competence-based training is more effective than other types of training, based on time or the number of repetitions, we moved students through all exercises taking into account our perception of their competence [21].

All trainees went through the same regimen of five basic laparoscopic drills:

1. Single-hand coordination: typical single hand small item transfer exercise.

2. Hand-to-hand coordination: hand-to-hand color marked rope transfer.

3. Working the angles: putting metal rings on appropriate rods positioned at different angles.

4. Precision and power control: precise positioning of light objects.

5. Camera angulation: forced utilization of 30-degree laparoscopic camera angulation for a basic laparoscopic drill.

The amount of training the students required to achieve acceptable performance of each task varied. They were allowed to proceed to further exercises when the performance on the previous one was satisfactory. We did not measure the time needed for each of the exercises but recorded the total training time. After the training was completed, students repeated the knot-tying 15-minute exercise.

For analytical purposes, students were split into two groups based on the number of knots tied during the second measurement. Those who tied more than 15 knots formed group $A$ and those who tied less than 15 knots formed group B. Post-training sEMG signals were compared between the groups.

The EMG data pre-processing procedure included the removal of the recordings that could have been associated with the signal's artifacts.

\section{Statistical analysis}

All data were analyzed using Statistica v.13 (StatSoft) and Python Pandas Package. The results are presented as mean \pm standard error of the mean, median and interquartile range when appropriate. The Shapiro-Wilk test was used to check for normality of data distribution. The non-parametric Wilcoxon signed-rank test and Mann-Whitney $U$ tests were chosen for non-normally distributed quantitative-dependent and independent variables.

The relationship between the number of knots tied and muscle activity was examined using the Spearman correlation. Results were considered statistically significant when the $p$-value was found to be less than 0.05 .

\section{Results}

Signal pre-processing procedures resulted in approximately $14 \mathrm{~min}$ of recording (6,500 data points) per subject per measurement for each of the eight electrodes. A total of 160 sEMG signals were recorded for a total of over 1.1 million data points. Fifteen out of 160 (9.4\%) recorded EMG signals were excluded from the analysis due to identified measurement errors caused by the disturbed electrode-skin contact during the movements.

The values of the medians of the registered signals' amplitude were taken into consideration in comparison to the pre-post activities of the individual muscles. The results are summarized in Figure 1, which presents the box plot of the respective muscle groups registered before and after laparoscopic training. The upper panels characterize the dominant hand, whereas the lower graphs are assigned to the non-dominant hand.

The highest values of the median signal amplitude among the examined muscles are manifested by the thenar muscle group, both before and after the training.

Significant decreases in the median values after the training period are visible for the non-dominant hand deltoid and trapezius muscles. In contrast, greater activities characterize the non-dominant thenar after a series of training.

Comparing pre- and post-training activity on the dominant side corresponding muscle groups does not show such a clear change in activity as is visible for the non-dominant side muscles.

Dissimilarity of the activation patterns between dominant and non-dominant hands can be observed, especially before the training. They largely disappear after the training, and the activation patterns of the 

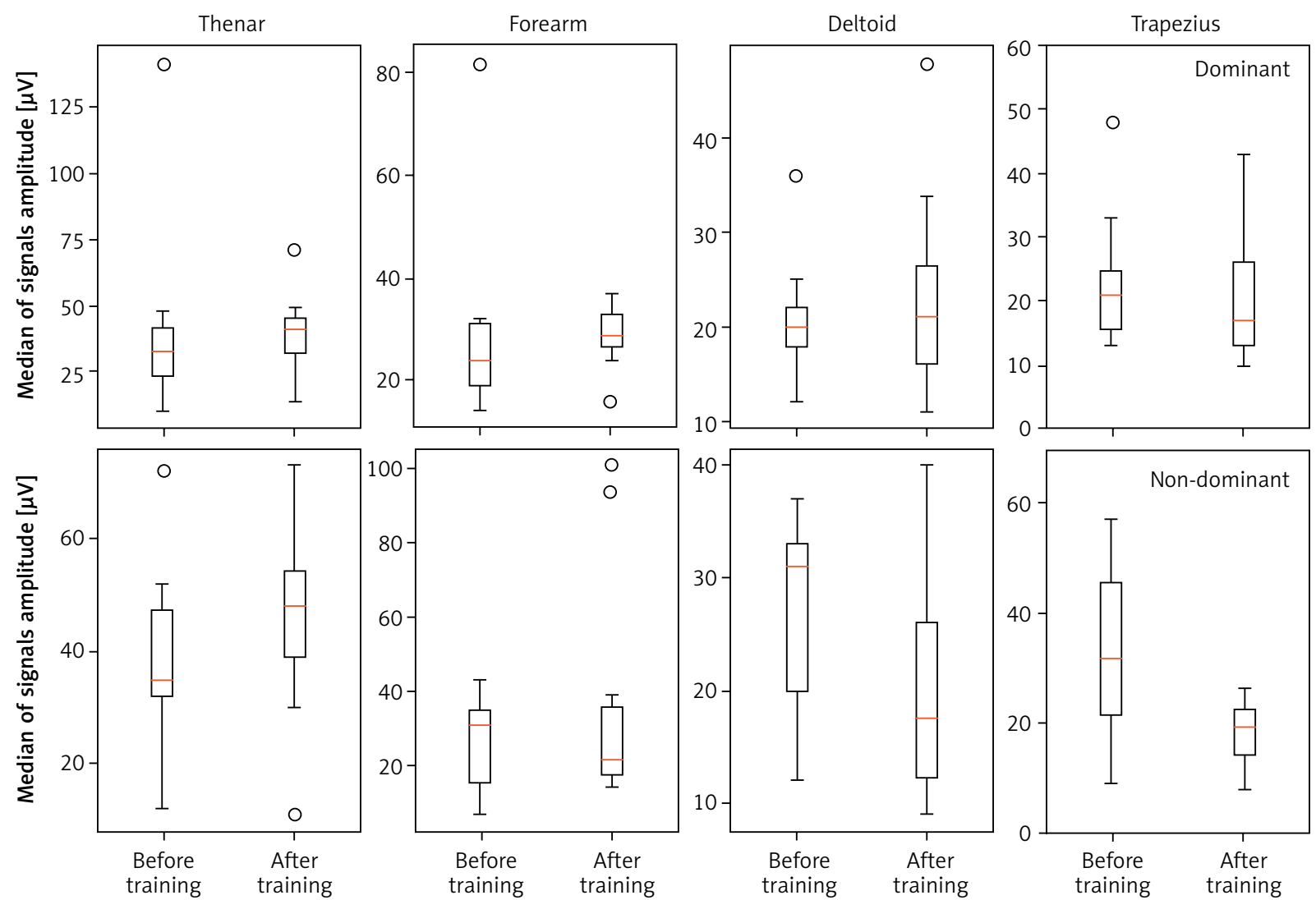

Figure 1. Box plot of respective muscle groups registered before and after laparoscopic training. Upper panels characterize the dominant hand, whereas the lower graphs are assigned to the non-dominant activity

dominant and non-dominant hand look much more alike after the training.

The average number of knots tied during the first contact with the laparoscopic tools was 1.7 (with half of the participants not able to tie any). After the sequence of training, this value grew to $14 \pm 4.65$ (56 s per knot).

For a more complete assessment of the association of muscle activity with laparoscopic training, the group of examined students was divided based on the number of tied knots. The criterion of the division was the ability to tie a number of knots greater than or equal to 15 . According to that criterion, a group of 6 students with higher competence after the training ( $>1 \mathrm{knot} / \mathrm{min})$ and 4 with lower $(<1 \mathrm{knot} /$ min) were selected.

Figure 2 shows an example of changes in activation patterns for two of the participants. The muscle activity of the student with the lowest number of tied knots (left panel) is higher than that of the student with the greatest progress after the training. This effect is visible for both the pre- and post-training stage.
The results of non-parametric Spearman correlation coefficients between muscle activities registered after the training and the number of tied knots are summarized in Table I.

They largely disappear after the training. With the exceptions of the dominant forearm and non-dominant thenar, the remaining muscles indicate a negative correlation between their activities and the level of laparoscopic skills measured by the number of correctly tied knots. The strongest relationship occurs for the non-dominant deltoid $(r=-0.863$, $p<0.05)$. Figure 3 presents a scatter-plot of the correlation coefficients calculated between the number of knots tied after the training and the activity of this muscle group. A relatively strong anticorrelation was also present in the non-dominant trapezius and forearm muscles $(r=-0.587, r=-0.504)$ (Table II).

In regard to the strength of the relationships between the number of knots and muscle activity (Table I), the groups of muscles that indicate the highest values of calculated autocorrelations $\left(R_{\text {spearman }}>0.5\right)$ were taken into consideration in further analysis. 


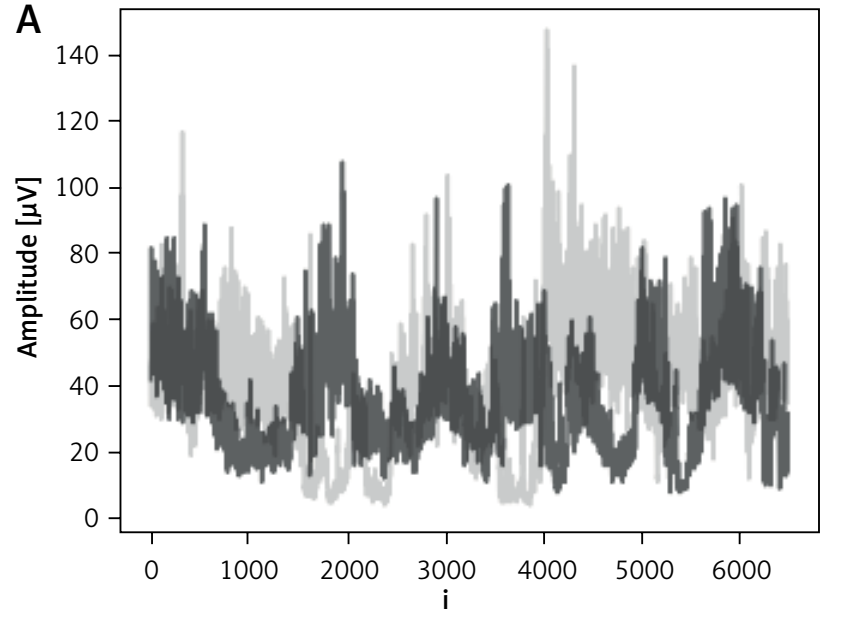

- After training

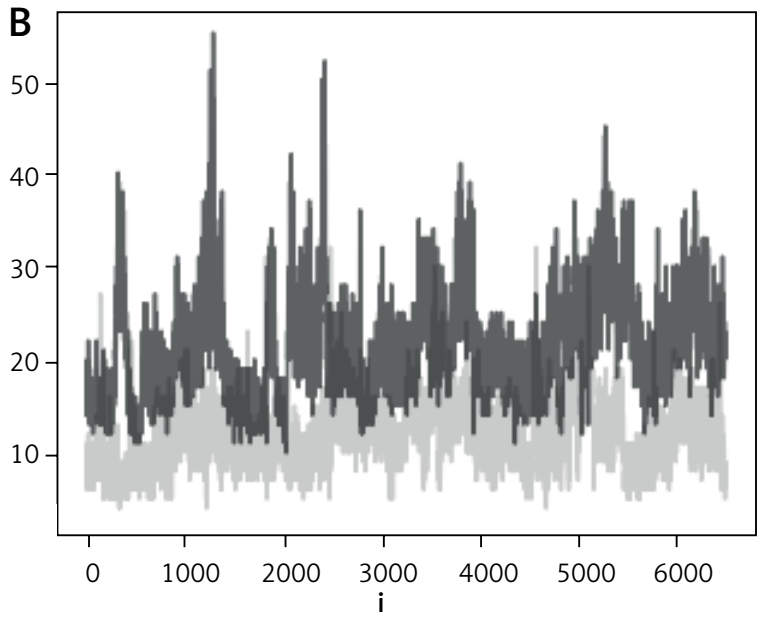

- Before training

Figure 2. Deltoid muscle activities recorded from two female subjects: A - the person with the lowest progress ( 3 tied knots before vs. 7 after the training); $\mathbf{B}$ - the person with the greatest improvement in the number of tied knots ( 0 before vs. 20 after the training)

Table I. Basic measurements of muscle activity recorded before and after training. All values in microvolts

\begin{tabular}{|c|c|c|c|c|c|c|c|c|c|}
\hline \multirow[t]{2}{*}{ Variable } & \multirow[t]{2}{*}{ Muscle } & \multicolumn{4}{|c|}{ Before training } & \multicolumn{4}{|c|}{ After training } \\
\hline & & Thenar & Forearm & Deltoid & Trapezius & Thenar & Forearm & Deltoid & Trapezius \\
\hline \multirow[t]{2}{*}{$\begin{array}{l}\text { Dominant } \\
\text { hand }\end{array}$} & $\begin{array}{l}\text { Mean } \\
\text { SE }\end{array}$ & $\begin{array}{c}48.37 \\
\pm 13.97\end{array}$ & $\begin{array}{r}33.13 \\
\pm 6.69\end{array}$ & $\begin{array}{l}22.32 \\
\pm 2.21\end{array}$ & $\begin{array}{l}24.15 \\
\pm 3.31\end{array}$ & $\begin{array}{l}48.57 \\
\pm 8.77\end{array}$ & $\begin{array}{l}32.94 \\
\pm 2.21\end{array}$ & $\begin{array}{l}24.04 \\
\pm 3.84\end{array}$ & $\begin{array}{l}22.59 \\
\pm 4.10\end{array}$ \\
\hline & $\begin{array}{l}\text { Median } \\
(\mathrm{IQR})\end{array}$ & $\begin{array}{c}33 \\
(15-62)\end{array}$ & $\begin{array}{c}24 \\
(14-36)\end{array}$ & $\begin{array}{c}20 \\
(15-29)\end{array}$ & $\begin{array}{c}21 \\
(13-31)\end{array}$ & $\begin{array}{c}41 \\
(25-72)\end{array}$ & $\begin{array}{c}29 \\
(19-40)\end{array}$ & $\begin{array}{c}21 \\
(12-35)\end{array}$ & $\begin{array}{c}17 \\
(11-32)\end{array}$ \\
\hline \multirow[t]{2}{*}{$\begin{array}{l}\text { Non-domi- } \\
\text { nant hand }\end{array}$} & $\begin{array}{l}\text { Mean } \\
\text { SE }\end{array}$ & $\begin{array}{l}42.41 \\
\pm 7.29\end{array}$ & $\begin{array}{l}28.83 \\
\pm 3.97\end{array}$ & $\begin{array}{l}28.23 \\
\pm 3.24\end{array}$ & $\begin{array}{l}34.47 \\
\pm 6.36\end{array}$ & $\begin{array}{l}50.67 \\
\pm 6.27\end{array}$ & $\begin{array}{r}38.49 \\
\pm 10.14\end{array}$ & $\begin{array}{l}21.24 \\
\pm 3.42\end{array}$ & $\begin{array}{l}19.09 \\
\pm 2.11\end{array}$ \\
\hline & $\begin{array}{l}\text { Median } \\
(\mathrm{IQR})\end{array}$ & $\begin{array}{c}35 \\
(23-68)\end{array}$ & $\begin{array}{c}31 \\
(13-47)\end{array}$ & $\begin{array}{c}31 \\
(13-46)\end{array}$ & $\begin{array}{c}32 \\
(15-56)\end{array}$ & $\begin{array}{c}48 \\
(27-72)\end{array}$ & $\begin{array}{c}22 \\
(14-48)\end{array}$ & $\begin{array}{c}17 \\
(9-37)\end{array}$ & $\begin{array}{c}19 \\
(10-27)\end{array}$ \\
\hline
\end{tabular}

The comparison of the left deltoid, left trapezius and left forearm with relationship to the average number of tied knots is presented in Figure 4.

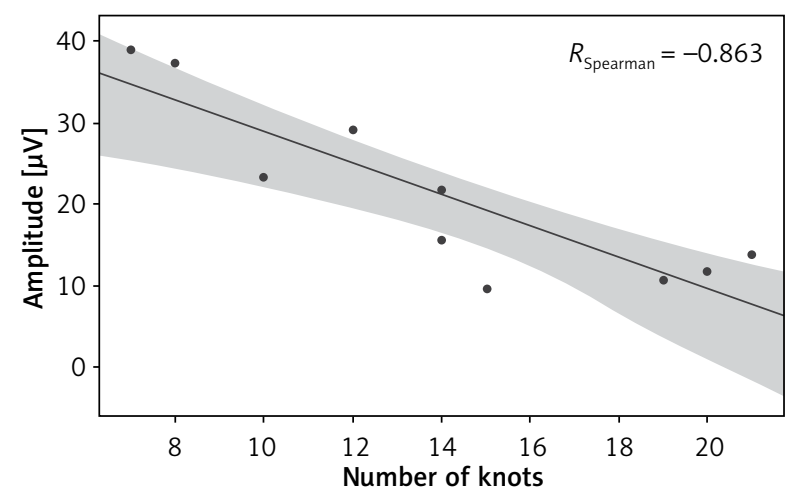

Figure 3. Scatter-plot of non-dominant hand deltoid muscle activity in relationship to number of tied knots after training
The most evident differences between the compared groups are visible in the case of the left deltoid. The mean activity after the training significantly decreased in the group of students with greater efficiency of the performed task (above 1 knot per minute), contrary to the students with the lowest progress after training.

Statistically significant differences measured with the Mann-Whitney $U$ test were observed between the post-activity of these two groups ( $p=0.014)$.

Despite the relatively strong anticorrelation $(r=-0.587)$, this effect was not observed for the left trapezius, where both groups were characterized by lower activity after the training. For the non-dominant forearm, an increase of mean activities after the series of training sessions was observed. 

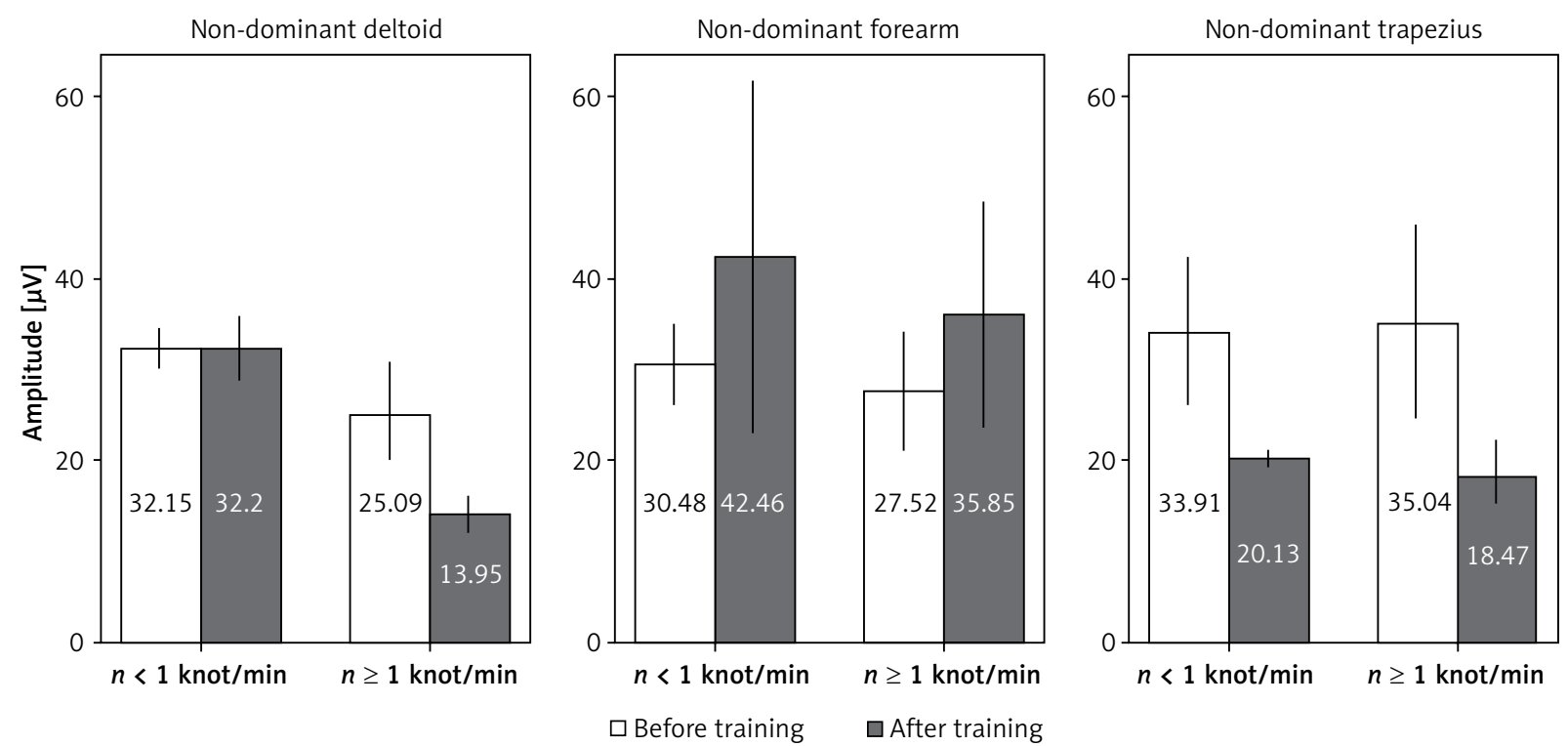

Figure 4. Relationship between amplitude of SEMG signal before and after training in low (white) and high-performance (gray) groups for selected muscle groups

Table II. Spearman correlation coefficients calculated between mean activities of the individual muscle group and number of tied knots after training

\begin{tabular}{|lcccccccc|}
\hline Parameter & \multicolumn{4}{c}{ Dominant hand } & \multicolumn{3}{c|}{ Non-dominant hand } \\
\hline Muscle & Thenar & Forearm & Deltoid & Trapezius & Thenar & Forearm & Deltoid & Trapezius \\
\hline$R_{\text {Spearman }}$ & -0.150 & 0.276 & -0.286 & -0.435 & 0.167 & -0.504 & $-0.863^{*}$ & -0.587 \\
\hline
\end{tabular}

\section{Discussion}

The significantly lower amplitudes after the series of training sessions observed for the non-dominant deltoid and trapezius may be the consequence of many factors, including better control over the mastered movements, an increase of general motor coordination, easier manipulation of laparoscopic tools, and also a smaller force put into the complex motor task [23]. The thenar muscle group and non-dominant forearm are characterized by increases of post-training activities. This might be the result of a proper utilization of the long wrist and finger flexors (mainly the brachialis, brachioradialis, palmaris longus and flexors: digitorum superficialis, carpi ulnaris and radialis), and in our opinion might represent proper muscular activation patterns developed with training.

It is difficult to assess the pre-post training changes in the activity of these muscle groups with equal accuracy due to the inconsistent results of the average and median amplitude, recorded from the non-dominant forearm. This effect is largely caused by the specific character of non-dominant forearm working dynamics. The majority of subjects have frequently occurring outliers that cannot be qualified as signal artifacts and they seem to be part of normal muscle activity.

The smaller changes of the dominant hand signal's amplitude after the training may indicate the greater involvement of this hand in the performed task. It may also indicate that training influenced the non-dominant hand skill acquisition more than the dominant hand [24]. Considering the possibilities of so-called cross-education (increased skill/strength in the contralateral muscle of the same group as that being trained), one may also speculate that despite lower utilization of the non-dominant hand, actual training gains are distributed more evenly [25].

Interesting results also concern the investigation of the dominant and non-dominant hand individually in the pre- and post-training stages. The relatively high disproportion in the activity of the trapezius 
muscle and thenar muscle group bilaterally can be observed only before training. One of the possible interpretations is that this is a sign of greater motor coordination of these muscle groups after the laparoscopic exercises. The greatest difficulties in the proper interpretation of the differences between the median activities are present for the thenar eminence. The standard error of the mean of activity of those muscles is the highest, making interpretations very prone to error. This finding has many potential reasons. The inter-electrode distance with concentric electrodes that we used is fixed. The amplitude of the signal also depends on that distance, and in proportion to the size of the muscle that distance is relatively high. Even a small change in the positioning of the electrode in relationship to the innervation zone of a respective muscle might change not only the amplitude but also the direction of the signal vector. The size of the muscle itself also has large variability, especially between male and female subjects [26]. Also, the direct contact of the electrodes with the laparoscopic instrument and semi-closed hand position may result in sweating where the electrode is located. This can have a significant impact on the measurement due to changes in the conductivity of the skin, and this is not easily avoided.

Valuable information is also provided by the comparison of the mean muscle activity and the number of tied knots (as a rough measure of the level of acquired competence). Our study confirmed an observation that muscular tension of the selected muscle group with special emphasis on the non-dominant deltoid is reduced with the increased level of manual competence, and that observation is also included in the OSATS questionnaire and was validated within the questionnaire several times for different fields of surgery $[27,28]$. Tension of the upper part of the limb (especially the shoulder and neck) is not only common in people performing precise, difficult tasks especially with external pressure (time, result), but is also considered a marker of stress [29]. According to this theory, diminished activity levels at proximal measurement points may indicate increased confidence but also increased proficiency in the task at hand. Increased activation of thenar muscles might indicate more active utilization of the dominant hand.

The main limitations of our study lie in the relatively small number of examined subjects and large variability among the recorded muscle activities. However, this is, at least to our knowledge, the first study on application of this methodology for that purpose, so it could also be considered a feasibility study. A larger study with a more refined protocol is certainly fully justified now that we know some changes are visible even at this level.

Another important limitation is that the only standardization of the instructors' judgment on the rate of progress of our trainees comes from teaching together over 800 students in the course of approximately 40 laparoscopic courses (10 h each) over the period of 3 years. There was no formal standard setting exercise, so it is theoretically possible that the progress rate was different for different instructors.

Obviously, because of the inter-subject variability associated with the different level of muscle response to fatigue, the muscle activity itself cannot be considered as a factor differentiating the students due to the level of the acquired skills. The most important issue concerns the changes in those activities after the sequence of training. As depicted in Figure 2, a better trained person is characterized by lower activity of the non-dominant deltoid over the entire length of the measurement (Figure 2 B). The opposite situation occurs for the student with the smallest progress, where the post-training activity in some parts significantly exceeds the amplitude of the first recording (Figure $2 \mathrm{~A}$ ). We hope that with a larger sample and more rigid protocol, the findings will one day also be confirmed for other muscle groups.

\section{Conclusions}

From the data analysis, we can safely conclude that at least for some muscle groups there is a change in activation patterns after laparoscopic training. In general, proximal muscle groups tend to become more relaxed and the distal ones become more active. Changes in the non-dominant hand are more pronounced than those in the dominant hand.

\section{Conflict of interest}

The authors declare no conflict of interest.

\section{References}

1. Jin SH, Kim DY, Kim H, et al. Multidimensional learning curve in laparoscopy-assisted gastrectomy for early gastric cancer. Surg Endosc 2007; 21: 28-33.

2. Major P, Wysocki M, Dworak J, et al. Analysis of laparoscopic sleeve gastrectomy learning curve and its influence on proce- 
dure safety and perioperative complications. Obes Surg 2018; 28: 1672-80.

3. Schlachta CM, Mamazza J, Seshadri PA, et al. Defining a learning curve for laparoscopic colorectal resections. Dis Colon Rectum 2001; 44: 217-22.

4. Feldman LS, Cao J, Andalib A, et al. A method to characterize the learning curve for performance of a fundamental laparoscopic simulator task: defining "learning plateau" and "learning rate". Surgery 2009; 146: 381-6.

5. Lee YH, Chong GO, Kim MJ, et al. Overcoming the learning curve of single-port total laparoscopic hysterectomy with barbed suture: a single surgeon's initial experience. Videosurgery Miniinv 2017; 12: 264-70.

6. Kwiatkowski AP, Stępińska G, Stanowski E, Paśnik K. Implementation of laparoscopic approach in colorectal surgery - a single center's experience. Videosurgery Miniinv 2018; 13: 27-32.

7. Alfa-Wali M, Osaghae S. Practice, training and safety of laparoscopic surgery in low and middle-income countries. World J Gastrointest Surg 2017; 9: 13-8.

8. Bashankaev B, Baido S, Wexner SD. Review of available methods of simulation training to facilitate surgical education. Surg Endosc 2011; 25: 28-35.

9. Hur HC, Arden D, Dodge LE, et al. Fundamentals of laparoscopic surgery: a surgical skills assessment tool in gynecology. JSLS 2011; 15: 21-6.

10. Budziński R, Michalik M, Frask A. Education in laparoscopic surgery. Videosurgery Miniinv 2008; 3: 22-9.

11. Zendejas B, Ruparel RK, Cook DA. Validity evidence for the fundamentals of laparoscopic surgery (FLS) program as an assessment tool: a systematic review. Surg Endosc 2016; 30: 512-20.

12. Laski D, Stefaniak TJ, Makarewicz W, et al. Structuralized boxtrainer laparoscopic training significantly improves performance in complex virtual reality laparoscopic tasks. Videosurgery Miniinv 2012; 7: 27-32.

13. Aggarwal R, Moorthy K, Darzi A. Laparoscopic skills training and assessment. Br J Surg 2004; 91: 1549-58.

14. Lefaucheur JP. Excitability of the motor cortical representation of the external anal sphincter. Exp Brain Res 2005; 160: 268-72.

15. Hackett L, Reed D, Halaki M, Ginn KA. Assessing the validity of surface electromyography for recording muscle activation patterns from serratus anterior. J Electromyogr Kinesiol 2014; 24: 221-7.

16. Mohseni Bandpei MA, Rahmani N, Majdoleslam B, et al. Reliability of surface electromyography in the assessment of paraspinal muscle fatigue: an updated systematic review. J Manipulative Physiol Ther 2014; 37: 510-21.

17. Nowakowski M, Trybek P, Machura L. Correlation based analysis of sEMG signals during complex muscle activity. Feasibility study of new methodology. Folia Med Cracov 2017; 57: 41-52.

18. Ricard MD, Ugrinowitsch C, Parcell AC, et al. Effects of rate of force development on EMG amplitude and frequency. Int J Sports Med 2005; 26: 66-70.

19. Jamaluddin FN, Ahmad SA, Noor SB, et al. Amplitude and frequency changes in surface EMG of biceps femoris during five days Bruce Protocol treadmill test. Conf Proc IEEE Eng Med Biol Soc 2015; 2015: 6219-22.
20. Marateb HR, Farahi M, Rojas M, et al. Detection of multiple innervation zones from multi-channel surface EMG recordings with low signal-to-noise ratio using graph-cut segmentation. PLoS One 2016; 11: e0167954.

21. Van Sickle KRR, Ritter EM, Baghai M, et al. Prospective, randomized, double-blind trial of curriculum-based training for intracorporeal suturing and knot tying. I Am Coll Surg 2008; 207: 560-8.

22. Barbero M, Merletti R, Rainoldi A. Atlas of Muscle Innervation Zones - Understanding Surface Electromyography and Its Applications. Barbero M (ed.). Springer, Manno, Switzerland 2012.

23. Swaminathan R, Williams JM, Jones MD, Theobald PS. The prediction of neck extensor force using surface electromyography. J Back Musculoskelet Rehabil 2016; 29: 279-85.

24. Molinas CR, Binda MM, Campo R. Dominant hand, non-dominant hand, or both? The effect of pre-training in handeye coordination upon the learning curve of laparoscopic intra-corporeal knot tying. Gynecol Surg 2017; 14: 1-10.

25. Lee M, Carroll TJ. Cross education: possible mechanisms for the contralateral effects of unilateral resistance training. Sports Med 2007; 37: 1-14.

26. Young AJ, Hargrove LJ, Kuiken TA. The effects of electrode size and orientation on the sensitivity of myoelectric pattern recognition systems to electrode shift. IEEE Trans Biomed Eng 2011; 58: 2537-44

27. Martin JA, Regehr G, Reznick R, et al. Objective structured assessment of technical skill (OSATS) for surgical residents. $\mathrm{Br}$ J Surg 1997; 84: 273-8.

28. Argun OB, Chrouser K, Chauhan S, et al. Multi-institutional validation of an OSATS for the assessment of cystoscopic and ureteroscopic skills. J Urol 2015; 194: 1098-105.

29. Lundberg U, Dohns IE, Melin B, et al. Psychophysiological stress responses, muscle tension, and neck and shoulder pain among supermarket cashiers. J Occup Health Psychol 1999; 4: 245-55.

Received: 16.06 .2018 , accepted: 2.09.2018. 